\title{
Violência e identificação de raça como consequência da categorização de grupo
}

\author{
Diego Macedo Gonçalves \\ Faculdade de Ciências, Cultura e Extensão do Rio Grande do Norte \\ Faculdade Natalense para o Desenvolvimento do Rio Grande do Norte
}

\begin{abstract}
Resumo
A Psicologia Evolucionista vem mostrando que a agressão coletiva direcionada aos membros de grupos adversários faz parte do repertório comportamental humano. A mente humana contém mecanismos desenhados para a formação de coalizões. Um desses mecanismos psicológicos, chamado de "Nós versus Eles", leva os indivíduos a se colocarem numa postura contrária ou favorável diante de um grupo, podendo gerar conflitos violentos. Uma das formas pela qual esse processo mental se manifesta é através da categorização de raça. $\mathrm{O}$ ser humano usa diversas pistas para categorizar grupos, entre elas, a cor da pele. Esse é um processo flexível e dinâmico que pode mudar dependendo do contexto social.

Palavras-chave: raça; categorização; coalizão; psicologia evolucionista; evolução.
\end{abstract}

\begin{abstract}
Violence and race identification as a consequence of group categorization. Evolutionary Psychology has shown that collective aggression toward out-group members in some specific situations, is part of the human behavioral repertoire. The human mind contains mechanisms designed for the formation of alliances and coalitions. This psychological mechanism, called "Us versus Them", influences individuals to assume a contrary or a favorable attitude in relation to a given group and may generate violent conflicts. One way whereby this mental process is manifested is through race categorization. The human being uses various cues to categorize groups, among them skin color. This is a plastic and dynamic process, which can change depending on the social context.
\end{abstract}

Keywords: race; categorization; coalition; evolutionary psychology; evolution.

$\mathrm{O}$ $\mathrm{s}$ motivos que levam o ser humano a expressar comportamento violento sempre foram tema de interesse da ciência. A tradicional psicologia social, por exemplo, pesquisa como os processos individuais podem levar à manifestação da atração, hostilidade e agressão em situações grupais (Brewer \& Kramer, 1985). Uma das causas para tanto interesse são os índices alarmantes que indicam o aumento do comportamento agressivo na atualidade (Lessa, 2004). O comportamento violento tem sido visto como um problema de saúde pública em nível nacional e internacional (Pino \& Werlang, 2006). Nos Estados Unidos, por exemplo, a violência interpessoal é a principal causa de mortes entre adultos jovens (Cornwell et al., 1995). Já no Brasil, mais de cinqüenta mil pessoas morreram vítimas de agressão só no ano de 2003 (Souza \& Lima, 2007). Os números despertam, na população geral, emoções como medo e insegurança e, conseqüentemente, maior interesse na elucidação dos problemas de segurança.

Agressão física, preconceito, agressão verbal e conflitos entre grupos - como a guerra - são formas conhecidas de violência que fazem parte do repertório comportamental humano.
Essa última é considerada a forma de violência mais contundente já que nenhuma outra modalidade apresenta um número tão expressivo de mortos. Ruanda, Kosovo e o Iraque são exemplos contemporâneos desse quadro, com mais de um milhão de mortos, somados os três conflitos. Além disso, só no século XX, mais de 160 milhões de pessoas morreram em decorrência de guerras, do genocídio e da opressão política (de Wall, 2007).

Freqüentemente, as causas desses problemas são atribuídas a questões contemporâneas, como influências sociais, culturais e à disparidade econômica na população. A forte ênfase nessa perspectiva mantém a esperança de que os índices de violência possam ser atenuados (Minayo, 1994). Políticas públicas são desenvolvidas com o intuito de amenizar essa situação. No entanto, observa-se que mesmo em menor freqüência ou com menor intensidade, o ser humano continua a realizar atos violentos.

Outros pesquisadores argumentam que há influência filogenética no comportamento agressivo, sugerindo que este comportamento pode ser parte integrante de nossa herança genética. Conflitos, violência e guerra fazem parte da história 
do ser humano, sendo tão antigos quanto à própria sociedade. Estão presentes em todas as culturas, e podem ser consideradas como uma característica universal humana (Queiroz, 2009).

De fato, os relatos do comportamento violento são muito antigos e parecem preceder até mesmo os registros escritos da história humana. Tooby e Cosmides (1988) argumentam, por exemplo, que a violência é um fenômeno que acompanha o homem desde o surgimento da espécie, constituindo um elemento natural da vida em sociedade.

\section{Relações entre a perspectiva evolucionista e o com- portamento agressivo}

A psicologia evolucionista é uma abordagem psicológica que usa conhecimentos da biologia evolutiva em pesquisas cujo intuito é investigar a estrutura da mente humana (Barkow, Cosmides, \& Tooby, 1992; Cosmides \& Tooby, 1997). Seu objetivo é provar que a estrutura psicológica, assim como a estrutura física, se desenvolveu a partir do processo de seleção natural. Ou seja, o objetivo é compreender como as pressões ambientais moldaram o cérebro humano ao longo das eras.

Um dos principais fundamentos da psicologia evolucionista é a proposição de que as atuais características humanas surgiram ao longo do passado evolutivo recente, aproximadamente durante as últimas mil a dez mil gerações (Hagen, 2002; Izar, 2009). Esse período é chamado de ambiente de adaptação evolutiva (AAE), e era um ambiente no qual o ser humano tinha que caçar, coletar, livrar-se de predadores e rivalizar com coalizões adversárias na disputa por recursos escassos (Gaulin \& MacBurney, 2001).

Por ser tratar de um ambiente marcado por situações limítrofes, somente os mais adaptados a esse ambiente sobreviveram e transmitiram seus genes. Os mecanismos psicológicos surgidos no decorrer desse processo possibilitaram aos nossos ancestrais encontrar a melhor saída para resolver problemas reprodutivos, tornando mais provável que eles deixassem um maior número de descendestes (Barkow et al., 1992; Cosmides \& Tooby, 1997; Tooby, Cosmides, \& Price, 2006). Na atualidade, o ser humano vive em contextos bem mais confortáveis e controlados, mas seus cérebros reproduzem características adaptadas às características do passado evolutivo. Entre elas estão o comportamento cooperativo, a escolha de parceiros, a linguagem, o processo de categorização de grupos e o comportamento violento em alguns contextos (Barkow et al., 1992). Se essas características estão presentes até os dias de hoje, é porque, provavelmente, os benefícios acarretados àqueles que as apresentaram promoveram uma vantagem evolutiva.

Essas conjecturas podem explicar uma ampla variedade de padrões comportamentais humanos, inclusive os socialmente não desejáveis como o comportamento violento. Há evidências, obtidas através de estudos paleontológicos, estudos comparativos com outros primatas antropóides e por estudos com sociedades tribais que vivem como caçadores-coletores, de que nossos ancestrais já agrediam outros da sua espécie em situações de conflitos (Wranghan \& Peterson, 1996).

Pesquisas osteológicas têm fornecido fortes evidências que nossos antepassados entravam em conflitos. Marcas de golpes encontradas em ossos de nossos ancestrais confirmam que houve episódios de agressão física desde a época dos Australopithecus (Lessa, 2004). As ossadas mostram ferimentos causados por projéteis ou por golpes produzidos por instrumentos rombudos. As freqüentes lesões existentes nas ossadas de membros das sociedades antigas apresentam padrões semelhantes aos encontrados nas populações mais modernas, mostrando uma continuidade do comportamento violento no decorrer do tempo. Isso pode ser um indício de que havia conflitos violentos no passado evolutivo. Lessa (2004) argumenta que desde o passado mais remoto, o homem tenta lidar com conflitos de interesses, poderes, valores, hábitos, mudanças e emoções inerentes ao ato de viver.

Evidências históricas também são fortes indícios de que o gênero Homo apresenta comportamento violento. Os livros de história estão repletos de relatos de conflitos bélicos entre diferentes etnias dando a impressão de que não parece ter havido espaço de tempo sem a existência de guerra em algum lugar deste planeta. Tooby e Cosmides (1988) argumentam que mesmo no AAE as sociedades de caçadores-coletores possivelmente competiam por alimentos, territórios e parceiras(os) sexuais com os membros das coalizões vizinhas e conseqüentemente entravam em conflitos.

Além disso, informações colhidas em praticamente todas as sociedades humanas parecem assegurar que em nenhuma delas se desconhece algum tipo de ação violenta (Queiroz, 2009). Arqueólogos e antropólogos argumentam que a violência era muito mais enraizada nas antigas sociedades de caçadorescoletores do que em épocas mais recentes (Keeley, 1996). Nas sociedades tribais modernas, por exemplo, as evidências mostram que eles estão em um constante estado de guerra. Dois terços dos caçadores-coletores modernos estão em um estado quase permanente de conflito tribal e cerca de $90 \%$ vão para a guerra pelo menos uma vez por ano. A taxa de mortes por guerras nessas populações é de $0,5 \%$ por ano, o que equivaleria a dois bilhões de pessoas morrendo durante o século XX. Mesmo entre populações tidas como pacíficas por pesquisas antropológicas encontrou-se indícios das mais diversas manifestações de violência (Wranghan \& Peterson, 1996).

Em muitas sociedades atuais de caçadores-coletores, o comportamento agressivo não só é executado freqüentemente como também é incentivado. Isso acontece, por exemplo, entre os Ianomâmis. Na Venezuela, os grupos atacam tribos rivais por causa de disputas territoriais ou por discussões aparentemente sem importância e, por fim, promovem emboscadas, geralmente, levando a homicídios. Os assassinos com mais mortes no seu histórico recebem status hierárquico destacado entre os membros da tribo e têm seu comportamento enaltecido pelos outros indivíduos (Wrangham \& Peterson, 1998).

Outras evidências foram obtidas através de estudos comparativos entre espécies. Etólogos vêm se empenhando na compreensão do comportamento de primatas não humanos. A idéia é que a ancestralidade comum recente permite estudar os comportamentos desses últimos como forma de entender o passado evolutivo do homem. Assim, esses estudos nos dão boas pistas sobre a história filogenética do comportamento violento.

Pesquisas usando métodos de observação com chimpanzés têm chegado à conclusão de que a guerra não é uma característica 
inerentemente humana (Manson \& Wrangham, 1991). Chimpanzés têm sido observados agindo de modo violento, apresentando conflitos por poder principalmente entre machos, realizando sangrentas disputas e até mesmo assassinatos (Wrangham \& Peterson, 1998). Algumas evidências sugerem que esses animais, além de tudo, são capazes de planejar emboscadas contra coalizões rivais (de Wall, 2007), o que indica a presença de uma grande capacidade de raciocínio lógico. Os chimpanzés caminham por entre as copas das árvores, silenciosamente, até chegar aos limites do território vizinho e atacam rapidamente sem possibilitar reação de suas vítimas. Eles, simplesmente, exterminam os membros das coalizões vizinhas de forma semelhante a que os homens fazem com seus inimigos, em circunstâncias de guerra (Wrangham \& Peterson, 1998).

As semelhanças podem indicar a disseminação da resposta violenta nas duas espécies (Manson \& Wrangham, 1991). É possível que um ancestral comum a ambas tenha difundido essa característica. Assim, o comportamento agressivo pode ter sido um atributo naturalmente selecionado no ambiente ancestral por ter beneficiado os sujeitos que o apresentavam. Mas vale salientar que, assim, como o ser humano se comporta violentamente em alguns contextos, ele também pode apresentar comportamento altruísta e empatia em outros contextos (de Wall, 2007).

Wilson e Daly (1985) utilizam explicações evolucionistas sobre o "comportamento agressivo" nas sociedades humanas contemporâneas. Eles mostram que os homens estão mais predispostos a cometer comportamento violento, agressão e até mesmo homicídio. Eles se envolvem em conflitos, matam e são vítimas de assassinato com maior freqüência do que as mulheres. Seus estudos ainda indicam que homens jovens se envolvem com maior freqüência em conflitos e situações de risco do que homens mais velhos, tendência que eles intitularam de "síndrome do macho jovem".

O período crítico para manifestação do comportamento violento coincide com a maturidade sexual do ser humano. Os jovens parecem se auto-afirmar através de envolvimento em situações hostis e de risco. Nesse período, competir por maior status dentro do seu grupo aumenta a possibilidade de relacionamento sexual, pois, apesar do elevado custo trazido pelo risco de ferimentos ou até mesmo de morte, os benefícios podem superá-los (Wilson \& Daly, 1985).

$\mathrm{Na}$ atualidade, entende-se que a violência humana sofre a influência de variáveis biológicas e sociais na sua manifestação, assim como uma grande parte do repertório comportamental humano. Se o ser humano apresenta alguma tendência para manifestar um comportamento específico ele também necessita, na mesma proporção, de um meio propício para sua manifestação. Estudos recentes de Wilson, Daly e Vasdev (2001) mostram que o fator mais importante no envolvimento de jovens machos em violência é a desigualdade social. Essa informação é dada através de análises pelo índice Gini de desigualdade de renda. Nesse índice o "0" representa a igualdade absoluta (todos com a mesma renda) e "l" representa a desigualdade absoluta (uma pessoa ganha tudo). Esses pesquisadores encontraram uma correlação positiva entre o índice Gini e taxas de assassinatos. Dessa forma, quanto maior o índice Gini em uma dada população, maior a taxa de homicídio. Isso indica que jovens podem manifestar comportamento violento com maior freqüência quando o contexto social não possibilita seu desenvolvimento social e financeiro. Portanto, pode-se perceber que existe uma regulação do comportamento violento pelo contexto social.

Há várias evidências de que a violência faz parte do repertório comportamental humano e que se manifesta em alguns contextos. Nem o desenvolvimento da tecnologia, nem o uso de novos métodos educacionais e nem o atual acúmulo de recursos conseguiram eliminar o comportamento violento manifestado pelo ser humano. Até hoje, a maneira mais utilizada para combater a violência é a própria violência, ou ao menos a ameaça de sua ocorrência. Isso se reproduz nas forças policiais e militares. No entanto, o fato do homem se comportar violentamente em alguns contextos não impossibilita que medidas possam ser tomadas no intuito de amenizar ou abolir tal comportamento. Conhecer a natureza humana, os mecanismos que permitem que os seres humanos se engajem em situações de conflito e o contexto social no qual a violência se manifesta ajuda a traçar as melhores estratégias para combatê-la.

\section{Psicologia das coalizões}

Cosmides, Tooby e Kurzban (2003) defendem a idéia de que a agressão coletiva é resultante de funções psicológicas responsáveis pela formação de alianças e coalizões. Isso significa que alguns processos cognitivos complexos geram respostas emocionais e comportamentais direcionadas aos aliados e rivais ao se deparar com situações de hostilidade (Tooby \& Cosmides, 1997).

Pesquisas sobre etnocentrismo mostram que, diante de situações de conflitos de interesse entre alianças distintas, mecanismos psicológicos parecem se ativar aumentando a coesão grupal e a hostilidade ao grupo adversário. Os sujeitos tendem a ver o próprio grupo como superior e seus valores como universais, e ver o grupo de fora como inferior (Hammond \& Axelrod, 2006). Mais do que isso, o ser humano tende a ter uma visão de mundo na qual o seu próprio grupo é tomado como centro de tudo e todos os outros são avaliados através dos seus valores, modelos e definições do que é a existência (Rocha, 1984). Como conseqüência, esse fenômeno psicológico promove a cooperação entre os membros de uma coalizão e a hostilidade e até mesmo a agressão direcionada aos membros de fora do grupo (Brewer, 1999; Brewer \& Kramer, 1985; Kurzban, Tooby, \& Cosmides, 2001).

A hipótese é de que um mecanismo denominado de "Nós versus Eles" incite seres humanos a se organizar em grupos e classificar pessoas como pertencentes ou não ao grupo de referência. Hammond e Axelrod (2006) argumentam que os psicólogos evolucionistas mostram que essa é uma tendência universal. Para eles, se o comportamento se manifesta repetidamente por uma espécie é porque provavelmente tem uma base adaptativa (Tooby \& Cosmides, 1988). Isso quer dizer que durante o processo evolutivo esses aparatos psicológicos surgiram, ajudando a resolver problemas como trocas sociais e relações entre coalizões.

No AAE, nossos ancestrais provavelmente se depararam com situações nas quais precisaram competir por recursos escassos com outros grupos de sua espécie. Ao se agrupar e 
apresentar algum grau de comportamento agressivo nesses contextos específicos, vantagens adaptativas foram geradas fazendo com que esse atributo se disseminasse para as gerações posteriores, estando presente até a atualidade (Barkow et al., 1992). O discernimento entre amigos e inimigos, por exemplo, acontece ao se categorizar sujeitos em "Nós versus Eles". Vale salientar o argumento de Queiroz (2009) ao afirmar que postular a base inata para o comportamento agressivo faz sentido apenas em termos de probabilidade de que essa postura se manifestará em alguns ambientes específicos e não em todos os ambientes.

O ser humano utiliza diversas pistas sociais como referência para distinguir os aliados dos rivais. Características sociais, culturais e até características fenotípicas servem como marcos referenciais (Cosmides et al., 2003). Essas pistas são usadas para discriminar pertinência, e se manifestam através das vestimentas, dos comportamentos, das gírias e até mesmo a da cor da pele. Assim, diferenças entre membros de um grupo tendem a ser minimizadas, enquanto que diferenças intergrupo permanecem muito evidentes e ressaltadas (Taylor, Fiske, Etcoff, \& Ruderman, 1978).

Todo esse processo é seguido de percepções negativas e de sentimentos de hostilidade voltados para os rivais. Tajfel (1982) argumenta, por exemplo, que as conseqüências geradas pela interação entre grupos provocam uma antecipação da natureza da interação. Assim, os sujeitos podem sentir aversão instantânea pelos adversários em situação de competição e formam uma opinião desfavorável sobre eles, agindo de modo a negar-lhe recompensas mesmo que isso seja oneroso para o seu próprio grupo. Informações pejorativas podem ser espalhadas, diminuindo a possibilidade de cooperação entre grupos. Adjetivos como, egoístas, traiçoeiros, maus, manipuladores, dissimulados e falsos são normalmente empregados para descrever os rivais.

Diante de contexto de conflito, o cérebro humano interpreta os cooperadores como aliados e os competidores como opositores gerando um fenômeno chamado de confiança despersonalizada. A identificação dos membros como pertencentes a sua coalizão promove uma confiança mútua e conseqüentemente o altruísmo condicional. Assim, as pessoas agem de forma cooperativa em função da associação ao grupo e independente das características de cada um (Brewer, 2001). Em situações de conflito, estereótipos podem promover avaliações distorcidas sobre as características e reputações dos membros de grupos rivais aumentando ainda mais a hostilidade já direcionada a eles.

Como conseqüência desses processos psicológicos, as interpretações do comportamento dos adversários geralmente são preconceituosas, errôneas e levam a julgamentos incorretos, mas todo tipo de argumento é usado para aumentar a hostilidade intergrupo. Tajfel (1982) afirma que as pessoas cristalizam suas impressões dos indivíduos para formar uma percepção de grupo como um todo. Parece vantajoso que membros de uma coalizão foquem nas características negativas dos adversários.

Apesar dos padrões perceptuais voltados aos adversários e aliados em situação de competição parecerem invariáveis, estudos realizados por psicólogos sociais afirmam que o contexto social pode ajudar na modificação desse padrão. Aronson, Blaney, Stephan, Sikes e Snapp (1978) enfatizam esse ponto de vista quando afirmam que interações positivas entre grupos podem reduzir a hostilidade inicial da categorização grupal. Diante de situação de conflito, ao se introduzir uma pista de atenuação de hostilidade (doação), a confiança direcionada ao grupo adversário pode aumentar.

Mas o contrário também é verdadeiro. Variáveis sociais podem precipitar o comportamento opositor. Condições econômicas desfavoráveis, calor e ruídos incômodos, por exemplo, podem gerar violência e conflitos entre grupos, no entanto não a determinam mecanicamente (McClain, 1993; Queiroz, 2009). Além disso, o potencial de conflito também pode ser multiplicado à medida que o grupo cresce, não somente pelo número adicional de participantes, mas também pela possibilidade da formação de facções e coalizões dentro do grupo inicial.

Todo esse processo, no entanto, só é possível se há um grupo coeso que possa partilhar esforços com o intuito de obter um denominador comum. Por isso, viver coletivamente implica viver em um ambiente onde o altruísmo recíproco mantém as relações entre os indivíduos. Choi e Bowles (2007) argumentam que no AAE o altruísmo dentro de uma coalizão pode ter levado a vitórias em conflitos dando acesso a recursos naturais/reprodutivos escassos, o que evidentemente contribuiu para o aumento da aptidão de seus membros. Os indivíduos de um mesmo grupo tendem a permanecer coesos e contrários ao grupo rival. Incitações à cooperação são dirigidas ao grupo de pertinência e visam aumentar a coesão do grupo. Dessa forma, torna-o mais forte na competição contra outros grupos (Yamamoto, 2008).

Nesse aspecto a cooperação é um importante requisito na manutenção das relações sociais (Alencar, 2008). Por muitas vezes, os indivíduos até mesmo se colocam em risco para ajudar seus aliados. Apesar de aparentemente isso implicar em custos para o cooperador e benefícios para quem recebe a ajuda (Macedo, 2007), o comportamento altruísta pode gerar possíveis recompensas futuras para o doador. Se essa estratégia comportamental pode gerar benefícios individuais mútuos, eles promovem vantagens evolutivas.

A Psicologia Evolucionista defende que essas características e mecanismos foram, provavelmente, moldados no nosso passado evolutivo. $\mathrm{O}$ aparato mental humano segrega o mundo em categorias, e a partir daí, maximiza os seus benefícios evolutivos, cooperando ou competindo. Todas essas descobertas são expressões de uma psicologia subjacente de formação de alianças e coalizões: um conjunto de programas neuro-cognitivos típicos da espécie que evoluiu para regular a cooperação dentro do grupo e o conflito entre coalizões rivais no ambiente de adaptação evolutiva (AAE) (Barkow et al., 1992; Cosmides et al., 2003; Kurzban et al., 2001; Tooby et al., 2006).

\section{Categorização de raça}

O processo de categorização pode levar a uma variedade de respostas comportamentais, entre elas a codificação de raças. Cosmides et al. (2003) explicam que a raça de um indivíduo pode ser percebida ou lembrada antes que um estereótipo racial possa ser ativado, e chamam essa percepção de identificação racial. Essa identificação, assim como outras pistas sociais, podem precipitar a categorização do tipo "Nós versus Eles". 
Pistas sociais podem ser utilizadas como critério para discriminar em favor do próprio grupo e contra grupos considerados rivais.

Mas, durante o século XX, pesquisas chegaram a conclusões diferentes sobre o processo de identificação racial. $\mathrm{O}$ ponto de partida dessas pesquisas foi dado por Gordon Allport em seu livro The nature of prejudice (Allport, 1954). A partir de então muitas pesquisas na área da psicologia social têm sido realizadas, confirmando seus estudos. Allport (1973) afirmava que percebemos melhor e mais rapidamente as características individuais semelhantes à nossa no que diz respeito a vários aspectos, tais como raça, sexo e idade. Esses estudos partem do ponto de vista de que a identificação de sexo, raça e idade são codificadas automaticamente e arbitrariamente.

Psicólogos evolucionistas refutam a idéia de que a identificação de raça seja automática (Cosmides et al., 2003; Kurzban et al., 2001). Para esses pesquisadores nenhuma parte dos mecanismos psicológicos do ser humano foi selecionada no decorrer do processo evolutivo especificamente para a codificação de raça. Uma vez que nossos ancestrais, no AAE, dificilmente se deslocavam a grandes distâncias, uma estrutura cerebral para identificação de raças não pode ter se desenvolvido, pois os indivíduos não tinham experiências com raças diferentes da sua. Assim, não poderia haver seleção para adaptações cognitivas desenvolvidas para codificar categorias raciais de forma preferencial, e menos ainda, codificá-las de maneira automática e obrigatória. Na verdade, a hipótese mais plausível é que a identificação racial seja um subproduto da identificação de alianças e coalizões (Kurzban et al., 2001).

Como já foi salientado, mecanismos que segregam grupos a partir de categorias sociais podem ser altamente voláteis, algumas vezes mudando no curso de minutos (Cosmides et al., 2003). Alianças mudam ao longo do tempo, variando em composição, pistas de superfície, duração e coesão interna. Por isso não há um único indicador de aliança (inclusive cor da pele) que deveria ser codificado uniformemente em todas as situações. Além disso, pistas arbitrárias (como características fenotípicas) só deveriam ter significância na medida em que adquirissem validade preditiva para a existência de uma coalizão. Na verdade, as pessoas parecem ter experiências que envolvem raça em suas vidas, e que predizem padrões de cooperação e conflito.

Kurzban et al. (2001) analisaram os efeitos da coalizão na codificação da raça. Seus resultados mostraram que as pessoas não codificam automaticamente a raça do indivíduo. Quando há outras pistas sociais para categorizar os grupos que não apenas a cor da pele, elas são utilizadas. Uma vez que o aparato cognitivo detecta a relação entre lealdade e aparência, dimensões estáveis de aparência que são comuns - e podem não ter significado em outras circunstâncias (por exemplo, vestuário, dialeto, postura) - podem emergir no sistema cognitivo como pistas fiéis de uma aliança.

Sob essas condições, o ser humano detecta as pistas compartilhadas - que podem ser a raça - e inferem alianças sociais. Assim, a mente mapeará raça como uma variável que indica as coalizões. De acordo com essas hipóteses a codificação de raça não é automática nem obrigatória (Cosmides et al., 2003).

A partir dos argumentos mencionados nesse artigo pode-se ter a impressão que o ser humano é um animal violento que não consegue controlar seus "instintos mais primitivos" e que é capaz de cometer homicídios em nome de benefícios evolutivos. No entanto, essa interpretação não corresponde ao ponto de vista do autor.

$\mathrm{Na}$ verdade, o que se quis demonstrar foi que o animal humano é capaz de cometer atos violentos diante de alguns contextos sociais e que essa capacidade pode ter conferido, ao longo da evolução humana, algum tipo de vantagem para os indivíduos que a expressavam. Mas na mesma proporção, sabe-se que o ser humano comporta-se de maneira extremamente generosa se o ambiente assim o permitir.

O cérebro humano é capaz de gerar emoções, comportamentos e processar informações. No entanto, essas atividades são reguladas de modo que se possa maximizar a aptidão, ou seja, garantir a sobrevivência e proporcionar a reprodução. Os mecanismos psicológicos indicam o comportamento mais apropriado segundo as circunstâncias ambientais (Cosmides \& Tooby, 1997). Isso significa que nossos mecanismos psicológicos devem avaliar adequadamente qual a melhor conduta a ser adotada diante de alguns estímulos específicos, seja esta uma conduta hostil ou uma conduta gentil.

Enquanto primata, o homem expressa violência. O maquinário neuropsicológico responde, sim, com sentimentos hostis diante de alguns estímulos aversivos. Mas, também, há mecanismos que possibilitam escolher outras alternativas.

Associar os ideais evolucionistas a condutas agressivas costuma assustar, por dar a entender que tal conduta não é passível de controle ou atenuação. Mas, o nosso ponto de vista é exatamente o oposto dessa conclusão. Assim como o ser humano possui mecanismos psicológicos que lhes possibilitam a destruição, também possuem outros mecanismos que lhes permitem gerar condutas gentis.

A abordagem da Psicologia Evolucionista pode levar a um entendimento cada vez maior da condição existencial humana e possibilitar o desenvolvimento de estratégias mais eficazes para o tratamento dos problemas humanos.

\section{Referências}

Allport, G. W. (1954). The nature of prejudice. Reading, Massachussets: Addison-Wesley.

Allport, G. W. (1973). Personalidade: padrões e desenvolvimento. São Paulo: Editora da Universidade de São Paulo.

Alencar, A. I. (2008). A cooperação em crianças da rede pública de Natal/R. uma abordagem evolucionista. Tese de doutorado, Universidade Federal do Rio Grande do Norte, Natal.

Aronson, E., Blaney, N., Stephan, C., Sikes, J., \& Snapp, M. (1978). The Jigsaw classroom. Beverly Hills: Sage.

Barkow, J. H., Cosmides, L., \& Tooby, J. (1992). The adapted mind: Evolutionary Psychology and the generation of culture. New York: Oxford University Press.

Brewer, M. B. (1999). The psychology of prejudice: ingroup love or outgroup hate? Journal of Social Issues, 55, 429-444.

Brewer, M. B. (2001). Ingroup identification and intergroup conflict: when does ingroup love become outgroup hate. In. R. D. Ashmore, L. Jussim \& D. Wilder (Orgs.), Social identify, intergroup conflict and conflict reduction (pp. 17-42): New York: Oxford University Press.

Brewer, M. B., \& Kramer, R. M. (1985). The psyhology of intergroup attitudes 
and behavior. Annual Review Psychology, 36, 219-243.

Choi, J-K, \& Bowles, S. (2006). The coevolution of parochial altruism and war. Science, 318, 636-640.

Cornwell, E. et al. (1995). National Medical Association Surgical Section position paper on violence prevention: a resolution of trauma surgeons caring for victims of violence. JAMA, 273, 1788-1789.

Cosmides, L., \& Tooby, J. (1997). Evolutionary Psychology: a primer. Disponível em http://www.psych.web.edu/research/cep/primer.html.

Cosmides, L., Tooby, J., \& Kurzban, R. (2003). Perceptions of race. TRENDS in Cognitive Sciences, 7, 173-179.

Gaulin, S. J. C., \& MacBurney, D. H. (2001). Psychology: an evolutionary approach. New Jersey: Practice Hall.

Hagen, E. H. (2002). The Evolutionary Psychology FAQ. Disponível em: http://www.anth.ucbs.edu/projects/human/epfaq/ep.html.

Hammond, R. A., \& Axelrod, R. (2006). The evolution of ethnocentrism. Journal of Conflict Resolution, 50, 926-936.

Izar, P. (2009). Ambiente de Adaptação Evolutiva. In E. Otta \& M. E. Yamamoto (Orgs.), Fundamentos da Psicologia:Psicologia Evolucionista (pp.22-31). Rio de Janeiro: Guanabara Koogan.

Lessa, A. (2004). Arqueologia da agressividade humana: a violência sob uma perspectiva paleoepidemiológica. História, Ciências, Saúde-Manguinhos, $11,279-296$.

Keeley L. H. (1996). War before civilization. New York: Oxford University Press.

Kurzban, R., Tooby, J., \& Cosmides, L. (2001). Can race be erased? Coalitional computation and social categorization. Proceedings of the Nacional Academy of Sciences of the United States of America, 98, 15387-15392.

Macedo, R. H. F. (2007). Cooperação animal. In: M. E. Yamamoto \& G. L. Volpato (Orgs.), Comportamento Animal (pp. 141-155). Natal: EDUFRN.

Manson, J. H., \& Wrangham, R. W. (1991). Intergroup aggression in chimpanzees and humans. Current Antrophology, 32, 369-390.

McClain, P. D. (1993). The changing dynamics of urban politics: black and hispanic municipal employment: is there competition? Journal of Politics, $55,399-414$
Minayo, M. C. S. (1994). Violência social sob a perspectiva da saúde pública. Cadernos de Saúde Pública, 10, 7-18.

Pino, V. D., \& Werlang B. S. G. (2006). Homicídio e lobo frontal: revisão da literatura. Interação em Psicologia, 127, 127-137.

Queiroz, R. S. (2009). Agressividade humana: contribuição da psicologia evolucionista e da antropologia. In. E. Otta \& M. E. Yamamoto (Orgs.), Fundamentos da Psicologia: Psicologia Evolucionista (pp. 127-132). Rio de Janeiro: Guanabara Koogan.

Rocha, E. (1984). O que é Etnocentrismo. São Paulo: Ed. Brasiliense.

Souza, E. R., \& Lima, M. L. C. (2007). Panorama da violência urbana no Brasil e suas capitais. Ciência \& Saúde Coletiva, 11, 1211-1222.

Tajfel, H. (1982). Social psychology of intergroup relations. Annual Reviews Psychology, 33, 1-39.

Taylor, S. E., Fiske, S. T., Etcoff, N. L., \& Ruderman, A. J. (1978). Categorical bases of person memory and stereotyping. Journal of Personality and Social Psychology, 36, 778-793.

Tooby, J., \& Cosmides, L. (1988). The evolution of war and its cognitive foundations. Comunicação apresentada em Evolution and Human Behavior Meetings, Michigan, USA.

Tooby, J.; Cosmides, L. \& Price. M. E. (2006). Cognitive adaptations for n-person exchange: the evolutionary roots of organizational behavior. Managerial and Decision Economics, 27, 01-27.

de Wall, F. (2007). Eu primata: por que somos como somos. (L. T. Mota, Trad.) São Paulo: Companhia das Letras.

Yamamoto, M. E. (2008). Porque somos como somos? A psicologia evolucionista e a natureza humana. Ciência Sempre, 4, 12-17.

Wilson, M., Daly, M., \& Vasdev, S. (2001). Income inequality and homicide rates in Canada and the United States. Canadian Journal of Criminology, 219-236.

Wilson, M., \& Daly, M. (1985). Ethology and Sociobiology. Competitiveness, risk taking, and violence: the young male syndrome, 6, 59-73.

Wrangham, R. \& Peterson, D. (1998). O Macho Demoníaco: as origens da agressividade humana. (M. H. C. Côrtes, Trad.) Rio de Janeiro: Objetiva.

Diego Macedo Gonçalves, doutor em Psicobiologia pela Universidade Federal do Rio Grande do Norte, é professor na Faculdade de Ciências, Cultura e Extensão do Rio Grande do Norte e Faculdade Natalense para o Desenvolvimento do Rio Grande do Norte. Endereço para correspondência: Av. dos Caiapós, 2885, Casa 56, CEP: 59067-400, Cidade Satélite, Natal-RN. Tel: (84)30822878/Cel: (84)88110553. E-mail: dimago. psico@gmail.com 